\title{
Sébastien Galtier
}

\section{Physique \\ de la Turbulence}

Des tourbillons aux ondes

SAVOIRS ACTUELS

EDP Sciences/CNRS Éditions 


\section{Dans la même collection}

Comprenons-nous vraiment la mécanique quantique? 2e édition

Franck Laloë

Mécanique Quantique - Tomes 1, 2 et 3 - Nouvelle édition

Claude Cohen-Tannoudji, Bernard Diu et Franck Laloë

La théorie statistique des champs

François David

Physique quantique, information et calcul

Pascal Degiovanni, Natacha Portier, Clément Cabart, Alexandre Feller et Benjamin Roussel

Le temps dans la géolocalisation par satellites

Pierre Spagnou et Sébastien Trilles

Retrouvez tous nos ouvrages et nos collections sur http://laboutique.edpsciences.fr

Illustration de couverture : Simulations numériques directes de la turbulence forte en hydrodynamique 2D (bas) et de la turbulence faible d'ondes gravitationnelles (haut). Sont montrées respectivement la vorticité signée et les fluctuations d'une composante de la métrique espace-temps.

Imprimé en France

(C) 2021, EDP Sciences, 17, avenue du Hoggar, BP 112, Parc d'activités de Courtabœuf, 91944 Les Ulis Cedex A et

CNRS Éditions, 15, rue Malebranche, 75005 Paris.

Tous droits de traduction, d'adaptation et de reproduction par tous procédés réservés pour tous pays. Toute reproduction ou représentation intégrale ou partielle, par quelque procédé que ce soit, des pages publiées dans le présent ouvrage, faite sans l'autorisation de l'éditeur est illicite et constitue une contrefaçon. Seules sont autorisées, d'une part, les reproductions strictement réservées à l'usage privé du copiste et non destinées à une utilisation collective, et d'autre part, les courtes citations justifiées par le caractère scientifique ou d'information de l'œuvre dans laquelle elles sont incorporées (art. L. 122-4, L. 122-5 et L. 335-2 du Code de la propriété intellectuelle). Des photocopies payantes peuvent être réalisées avec l'accord de l'éditeur. S'adresser au : Centre français d'exploitation du droit de copie, 3, rue Hautefeuille, 75006 Paris. Tél. : 0143269535.

EDP Sciences

ISBN (papier) : 978-2-7598-2535-6, ISBN (ebook) : 978-2-7598-2566-0

CNRS Éditions

ISBN (papier) : 978-2-271-13783-8, ISBN (ebook) : 978-2-271-13785-2 